\title{
Trayectorias educativas en Argentina: un análisis a partir del cálculo de medidas de flujos y niveles de escolarización, 1974-2009
}

\author{
Educational trends in Argentina: an analysis based on \\ measurements of flows and levels of schooling, 1974-2009
}

\author{
Dra. Mara Leticia Rojas \\ Universidad Nacional del Sur (UNS) y Consejo Nacional \\ de Investigaciones Científicas y Tecnológicas (CONICET)
}

Buenos Aires, Argentina mrojas@uns.edu.ar

Recibido: 20-X-2011 • Aceptado 9-XII-2011 • Corregido 14-XII-2011

\begin{abstract}
Resumen: En este artículo se proponen y analizan tanto medidas de niveles educativos (mediante la definición propia de las variables "cantidad de años de educación finalizados" $y$ "mayor nivel educativo obtenido") como de flujos educativos (por medio del cálculo de "tasas brutas" y "tasas netas de escolarización") a fin de observar su evolución reciente y realizar un perfil actualizado de la distribución de logros educacionales en la población argentina. A diferencia de la mayoría de la literatura, la utilización de la encuesta de hogares permite la realización de los indicadores de niveles y ciclos efectivamente finalizados, sin supuestos restrictivos adicionales tales como el promedio de años de repitencia o abandono del alumnado. Si bien la evolución de los flujos educativos en términos de tasas de matriculación ha sido favorable, llama la atención la ampliación de las brechas entre tasas brutas y netas, y la dependencia de dicha evolución de los vaivenes económicos. Además, el acervo educativo medido por niveles y años de enseñanza concluidos muestra dos puntos fuertes de concentración en la distribución a lo largo del espacio poblacional. Estas observaciones abren cuestionamientos acerca de la calidad y efectividad del stock educativo a futuro y de la polarización existente en la actualidad.
\end{abstract}

\section{Introducción}

La formulación de políticas educativas y la evaluación de sus posteriores resultados deberían basarse, al menos en parte, en el estudio del estado y evolución de los indicadores de enseñanza. En este sentido, uno de los puntos cruciales y de más difícil establecimiento es la correcta medición del acervo educativo de un país. Aunque las instituciones escolares reporten anualmente el alumnado total registrado, se requieren estimaciones poblacionales para calcular el grupo etario correspondiente y así obtener las tasas de matriculación habitualmente reportadas. Otro factor que debería considerarse es el fenómeno a ser identificado. Hay variables referidas a los niveles educativos, tales como la cantidad de años promedio de escolarización de la 
Palabras claves: Educación, niveles, flujos, Argentina, educativo

\begin{abstract}
In this paper they are proposed and analyzed measures of educational levels (by defining the variables "years of education" and "greater educational attainment") as well as educational flows (through the estimation of "gross" and "net enrollment rates") in order to observe their recent evolution and to make an updated profile of the distribution of educational attainment across the Argentinean population. Unlike most of the literature, the use of household survey allows the construction of educational indicators as levels and cycles actually finished without additional restrictive assumptions such as the average years of repetition or dropout. It will see that, although the flows evolution in terms of enrollment rates has been positive, there has been an enlargement of the gap between gross and net enrollment rate and they strongly depend on economic shocks. In addition, the stock measured by levels and years of education shows two points of concentration in the distribution across the population space. These observations open up questions about the quality and effectiveness of future educational stock and about the polarization existing today.
\end{abstract}

Keywords: Education, levels, flows, Argentina fuerza laboral en un momento del tiempo o la composición de la población por categoría educacional. Al mismo tiempo, existen variables que identifican flujos y que pueden dar una idea aproximada de la acumulación de conocimientos y habilidades de la población a futuro. Este es el caso de las tasas de matriculación reportadas para los diferentes grados de enseñanza.

Algunos de los primeros trabajos en realizar una aproximación de los niveles educativos de las sociedades fueron los de Psacharopoulos y Arriagada (1986, 1992) y Kyriacou (1991), quienes proveyeron una estimación de la medida de años de escolarización de la fuerza laboral para un grupo de países, en donde los años promedio de cada nivel educativo se utilizaban como variable aproximada de los años reales efectivamente realizados por los individuos en el sistema de escolarización. De la Fuente y Doménech (2006) suministraron información de la fracción de población de 25 años o más que había empezado (aunque no necesariamente concluido) cada nivel de educación. Barro y Lee (1993, 1996, 2000, 2010) y Cohen y Soto (2007) construyeron datos de educación combinando datos censales y tasas de matriculación para un grupo considerable de países, en intervalos de 5 años y para la población de 15 y 25 años o más. En el caso de datos faltantes, utilizaron interpolaciones tomando las observaciones de un quinquenio hacia atrás o hacia delante y el método de inventario permanente mediante el uso de tasas de matriculación y la composición por edades de la población.

La principal crítica a estos trabajos se funda en el hecho de que la cantidad de años completos de educación es calculada como un promedio de la cantidad de años invertidos en cada nivel educativo según la duración legal de los mismos en cada país, estableciéndose la diferencia entre niveles completos e incompletos de acuerdo con las tasas de finalización estimadas. Por otra parte, el método de inventario permanente comienza con las cifras de los censos tomadas como puntos de referencia 
de los niveles educativos, y luego usa las tasas de matriculación escolar de los diferentes grados a fin de estimar los cambios producidos en los mismos, realizando ajustes por tasas de mortalidad. Para el caso particular de las economías latinoamericanas, la utilización de tasas de matriculación y niveles educativos, antes que los años de escolarización efectivamente concluidos, podría arrojar resultados altamente sesgados dado el alto porcentaje de tasas de abandono, repitencia y extraedad escolar. Nehru, Swanson, y Dubey (1995) afirmaron que Barro y Lee (1993) y Psacharopoulos y Arriagada (1986) habían forzado el análisis al establecer como cantidad de años educativos el promedio de los niveles reportados. Aun cuando se corrijan por tasas de repetición o abandono, las estimaciones no están libres de errores al incluir supuestos tales como el número de veces que un individuo repite un curso. Asimismo, la transformación del número de años promedio de un nivel educativo a número de años concluidos en la escuela puede ser aún más imprecisa.

Por lo tanto, este trabajo pretende establecer ciertas medidas de educación para el caso de la República Argentina a partir del uso de la Encuesta Permanente de Hogares (período 1974-2009), con el objetivo de brindar una idea aproximada de cuál ha sido la evolución reciente de los flujos y niveles educativos, y establecer un perfil actualizado de la conformación de la población según el acervo de conocimientos y habilidades, asumiendo que los logros obtenidos a partir de la educación formal son una aproximación de dichos conceptos. Para esto, se presentan los indicadores tradicionales (niveles y tasas de escolarización) para el período comprendido entre 1974 y 2009, dado que la disponibilidad de datos comienza en 1974 y se procuran estimaciones quinquenales de acuerdo a la literatura habitual.

Para los fines propuestos, el trabajo se organiza de la siguiente forma. En el apartado II se resume la metodología utilizada. En el apartado III se introducen medidas de flujo y acumulación y, en el apartado IV, medidas de niveles o stocks educativos, con los análisis correspondientes. Por último, se presentan las consideraciones finales.

\section{Metodología y datos}

Los datos para el cálculo de tasas y niveles educativos fueron extraídos de la Encuesta Permanente de Hogares (EPH a partir de aquí), realizada por el Instituto Nacional de Estadísticas y Censos (INDEC) de la República Argentina ${ }^{1}$. Respecto de los indicadores agregados construidos por otros autores como Barro y Lee (2010), la utilización de la encuesta permite, a partir de 1998, el reconocimiento de los años exactos de educación formal que cada individuo posee, independientemente de los cambios en la legislación vigente y sin la necesidad de realizar supuestos acerca de la cantidad de años promedio que un nivel educativo representa, evitando, además, la contabilización a partir de las tasas de matriculación corregidas por tasas de abandono y repitencia. Esto también permite la visualización de niveles completos e incompletos sin el artilugio de recurrir a las tasas de finalización. Para el caso particular de este trabajo, la secuencia trimestral de la encuesta a partir del 2003 permite tomar de base los datos correspondientes al segundo trimestre de cada año, dado que la legislación argentina postula que cada cohorte corresponde a los individuos nacidos entre julio del año $t$ y junio del año $t+1$. Por lo tanto, los datos correspondientes a junio brindan datos de cohortes educativas completas. Para los años anteriores, se utilizaron las encuestas correspondientes a las ondas de los meses de mayo ${ }^{2}$.

Siguiendo la bibliografía tradicional, se tomaron observaciones quinquenales, dado que las modificaciones en acervo educativo suele ser el resultado de procesos de mediano plazo, y se analizaron los grupos 
etarios de 15 a 64 años de edad (población total en edad de trabajar) y de 25 a 64 años de edad.

Para el caso de las variables identificativas del flujo educacional, se calcularon las "tasas brutas de escolarización" y las "tasas netas de escolarización" para los diferentes niveles educativos ${ }^{3}$. La primera se entiende como el cociente entre el número de estudiantes matriculados en un nivel y el número de personas que deberían estar matriculados según el grupo etario correspondiente, mientras que el segundo indicador se define como el porcentaje de estudiantes matriculados en un nivel con la edad correspondiente a ese nivel y el número de personas que deberían estar efectivamente matriculados.

Como variables de niveles educativos se consideraron "mayor nivel educativo alcanzado" y "años de educación efectivamente finalizados" en el caso de los años con disponibilidad de datos. Esto es, la cantidad de años exactos de educación pudo ser calculada a partir de modificaciones realizadas en el cuestionario de la EPH en 1998 para los años 2004 y 2009. En el caso de 1999, el porcentaje de respuestas omitidas para este ítem fue relevante y el cálculo de "años efectivamente finalizados" resultaba inconsistente.

\section{Medidas de acumulación o flujo}

Formichella y Rojas (2007) analizaron desde una perspectiva institucional las modificaciones sufridas por el sistema educativo argentino durante las últimas cuatro décadas, a partir de los procesos de descentralización y la extensión de la escolaridad compulsiva, la cual llevó la obligatoriedad de 7 a 10 años en 1993 y, posteriormente, a 13 años en el 2006. Para dar una idea del avance en términos del incremento en la matriculación generado a partir de estas modificaciones, la Tabla 1 muestra la matrícula total y las variaciones interanuales acaecidas desde mediados de los noventa hasta la actualidad. El fuerte incremento del alumnado en el nivel secundario entre los años 1997 y 1998 responde a la llegada a dicho nivel de las cohortes insertas en el sistema educativo a partir de la Ley Federal de Educación de 1993. Lamentablemente, no se cuenta con una serie de mayor longitud para el total del país que muestre los incrementos iniciales (si los hubo) en el nivel primario ${ }^{4}$.

Lo anterior es coincidente con el incremento mostrado por las tasas brutas de matriculación en el sector secundario entre las observaciones de 1994 y 1999 en la Figura 1. En general, en el análisis de las variables correspondientes al flujo o acumulación de conocimientos, las tasas de escolarización tanto brutas como netas construidas a partir de la EPH muestran un incremento sustancial entre la década de 1970 e inicios del presente siglo. Este aumento fue particularmente notorio en los niveles secundario y superior, dado que para el primario las tasas de escolarización ya eran elevadas a inicios del período bajo estudio $^{5}$.

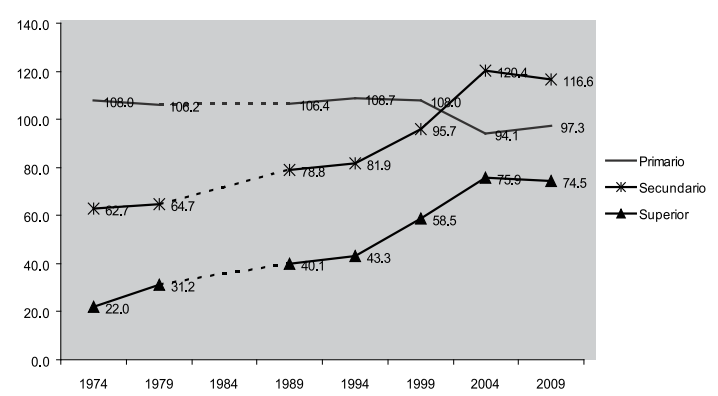

Figura $\mathrm{N}^{\circ}$ 1: Evolución de las tasas brutas de escolarización para todos los niveles

Fuente: Elaboración propia con base en datos de la EPH (INDEC). 
Tabla 1

Matrícula total y variaciones interanuales según nivel educativo ${ }^{(1)}$

\begin{tabular}{lcccccc}
\hline \multirow{2}{*}{ Año } & \multicolumn{2}{c}{$\begin{array}{c}\text { Total niveles primario y } \\
\text { secundario }\end{array}$} & \multicolumn{2}{c}{$\begin{array}{c}\text { Total nivel primario // EGB } \\
\text { 1 y } \text { (2) }^{2}\end{array}$} & \multicolumn{2}{c}{$\begin{array}{c}\text { Total nivel sec. // EGB 3 y } \\
\text { Polimodal (3) }\end{array}$} \\
\cline { 2 - 7 } & $\begin{array}{c}\text { Total } \\
\text { Matrícula }\end{array}$ & $\begin{array}{c}\text { Variación } \\
\text { Anual }\end{array}$ & $\begin{array}{c}\text { Total } \\
\text { Matrícula }\end{array}$ & $\begin{array}{c}\text { Variación } \\
\text { Anual }\end{array}$ & $\begin{array}{c}\text { Total } \\
\text { Matrícula }\end{array}$ & $\begin{array}{c}\text { V a r i a c i ó n } \\
\text { Anual }\end{array}$ \\
\hline 2010 & 8317091 & 0.66 & 4637463 & -0.13 & 3679628 & 1.67 \\
2009 & 8262661 & 0.92 & 4643430 & -0.44 & 3619231 & 2.73 \\
2008 & 8187157 & 0.95 & 4664025 & 0.39 & 3523132 & 1.69 \\
2007 & 8110372 & -1.78 & 4645843 & -1.69 & 3464529 & -1.89 \\
2006 & 8257334 & 2.50 & 4725915 & 2.80 & 3531419 & 2.10 \\
2005 & 8056031 & -0.87 & 4597404 & -1.06 & 3458627 & -0.61 \\
2004 & 8126642 & 0.29 & 4646779 & 0.56 & 3479863 & -0.07 \\
2003 & 8103106 & -1.30 & 4620916 & -2.09 & 3482190 & -0.24 \\
2002 & 8209804 & 0.31 & 4719335 & 0.43 & 3490469 & 0.14 \\
2001 & 8184782 & 1.20 & 4699128 & 0.67 & 3485654 & 1.92 \\
2000 & 8087907 & 2.51 & 4668006 & 1.29 & 3419901 & 4.22 \\
1999 & 7890021 & 1.13 & 4608509 & -0.04 & 3281512 & 2.82 \\
1998 & 7801815 & 2.43 & 4610376 & -10.53 & 3191439 & 29.54 \\
1997 & 7616864 & 2.48 & 5153256 & 2.15 & 2463608 & 3.19 \\
1996 & 7432301 &. & 5044827 & - & 2387474 &. \\
\hline
\end{tabular}

Fuente: Elaboración propia con base en datos de la Dirección Nacional de Información y Evaluación de la Calidad Educativa (DINIECE), Ministerio de Educación.

(1) Corresponde a educación común, excluyendo las modalidades de educación especial y educación para adultos.

(2) En el período $1996-2000$, este nivel incluye de $1^{\circ}$ a $7^{\circ}$ grado de la educación primaria y de $1^{\circ}$ a $6^{\circ}$ año de la Educación General Básica (EGB). A partir del 2001, corresponde a los años $1^{\circ}$ a $6^{\circ}$ de la educación primaria en completa correspondencia con los niveles EGB 1 y 2.

(3) En el período $1996-2000$, este nivel incluye de $1^{\circ}$ a $5^{\circ}$ y $6^{\circ}$ año de la educación secundaria, de $7^{\circ}$ a $9^{\circ}$ año de EGB 3 y de $1^{\circ}$ a $3^{\circ}$ de Polimodal. A partir del 2001, corresponde a los años $7^{\circ}$ a $12^{\circ}$ de estudio, en correspondencia con EGB 3 y Polimodal.

El incremento en el número de matriculados en la escuela secundaria fue más importante en el sistema de educación pública que en la de gestión privada, así como también fueron más abruptas y continuas las caídas en el primer sistema a partir del 2003, tanto en el nivel secundario como primario (ver Tabla 2). De hecho, el aumento en la matriculación, aunque moderado, había continuado hasta ese año en ambos niveles.
Luego del 2001 se vislumbra una desaceleración de la matrícula y un decrecimiento a partir del 2003 y hasta el año 2005 en los niveles de educación secundaria. A partir de entonces, la tendencia ha sido nuevamente positiva en el conjunto de la educación primaria y secundaria, fundamentalmente a causa del incremento en la matrícula de esta última. Los 8 millones de individuos en el sistema educativo en el 2009 representan más del $20 \%$ de la población argentina. 
Tabla 2

Matrícula total y variaciones interanuales según tipo de gestión y nivel educativo ${ }^{(1)}$

\begin{tabular}{rcccccccc}
\hline \multirow{2}{*}{ Año } & \multicolumn{3}{c}{ Total primario // EGB 1 y $2^{(2)}$} & \multicolumn{2}{c}{ Total secundario // EGB 3 y Polimodal ${ }^{(3)}$} \\
\cline { 2 - 8 } & \multicolumn{2}{c}{ Estatal } & \multicolumn{2}{c}{ Privado } & \multicolumn{2}{c}{ Estatal } & \multicolumn{2}{c}{ Privado } \\
\hline 2010 & 3484217 & -0.71 & 1153246 & 1.68 & 2657956 & 2.25 & 1021672 & 0.17 \\
2009 & 3509259 & -1.15 & 1134171 & 1.82 & 2599336 & 2.80 & 1019895 & 2.54 \\
2008 & 3550088 & -0.82 & 1113937 & 4.47 & 2528547 & 1.71 & 994585 & 1.64 \\
2007 & 3579528 & -2.00 & 1066315 & -0.64 & 2486005 & -1.90 & 978524 & -1.87 \\
2006 & 3652695 & 1.91 & 1073220 & 5.93 & 2534266 & 1.69 & 997153 & 3.18 \\
2005 & 3584266 & -2.19 & 1013138 & 3.15 & 2492211 & -1.13 & 966416 & 0.75 \\
2004 & 3664573 & -0.07 & 982206 & 3.00 & 2520686 & -0.79 & 959177 & 1.89 \\
2003 & 3667286 & -2.40 & 953630 & -0.84 & 2540767 & -0.37 & 941423 & 0.12 \\
2002 & 3757646 & 0.59 & 961689 & -0.18 & 2550133 & 0.21 & 940336 & -0.05 \\
2001 & 3735696 & 0.83 & 963432 & 0.04 & 2544841 & 1.78 & 940813 & 2.32 \\
2000 & 3704973 & 0.70 & 963033 & 3.64 & 2500413 & 3.75 & 919488 & 5.51 \\
1999 & 3679308 & 0.71 & 929201 & -2.91 & 2410047 & 3.56 & 871465 & 0.83 \\
1998 & 3653289 & -10.33 & 957087 & -11.32 & 2327190 & 31.85 & 864249 & 23.72 \\
1997 & 4074024 & 2.13 & 1079232 & 2.21 & 1765038 & 3.91 & 698570 & 1.41 \\
1996 & 3988973 &. & 1055854 &. & 1698619 &. & 688855 &. \\
\hline
\end{tabular}

Fuente: Elaboración propia con base en datos de la Dirección Nacional de Información y Evaluación de la Calidad Educativa (DINIECE), Ministerio de Educación.

(1) Corresponde a educación común, excluyendo las modalidades de educación especial y educación para adultos.

(2) En el período $1996-2000$, este nivel incluye de $1^{\circ}$ a $7^{\circ}$ grado de la educación primaria y de $1^{\circ}$ a $6^{\circ}$ año de la Educación General Básica (EGB). A partir del 2001, corresponde a los años $1^{\circ}$ a $6^{\circ}$ de la educación primaria en completa correspondencia con los niveles EGB 1 y 2.

(3) En el período $1996-2000$, este nivel incluye de $1^{\circ}$ a $5^{\circ}$ y $6^{\circ}$ año de la educación secundaria, de $7^{\circ}$ a $9^{\circ}$ año de EGB 3 y de $1^{\circ}$ a $3^{\circ}$ de Polimodal. A partir del 2001, corresponde a los años $7^{\circ}$ a $12^{\circ}$ de estudio, en correspondencia con EGB 3 y Polimodal.

Las variaciones en el número total de alumnos matriculados podrían deberse a dos cuestiones: las modificaciones en las tasas de escolarización y las variaciones en la composición por edades de la población. Analizando conjuntamente las series de datos, puede verse que, mientras el número de matriculados prácticamente no se vio modificado en el período 1999-2009, las tasas brutas de matriculación cayeron de forma evidente. Esto demuestra un mayor número de individuos en edad de escolarización primaria que no acceden a ese nivel. Este hecho es preocupante, en particular, a partir de la caída en las tasas de matriculación primaria luego de la crisis económica del 2001.

La matriculación se incrementó en un 6\% de 1999 al 2004 y en un 4\% desde entonces hasta el 2009; mientras que las tasas brutas de matriculación se vieron fuertemente incrementadas en el primer período pero levemente disminuidas en el segundo quinquenio.

La Figura 2 muestra que las tendencias de las tasas netas siguieron recorridos similares a las tasas brutas de escolarización, pero el incremento de las diferencias entre estos dos indicadores es de gran importancia, 
especialmente para los niveles secundario y superior a partir de los años noventa, llegando al 36.5 y $39 \%$, respectivamente, en el 2004 . Si bien la cobertura del sistema de educación secundaria ha llegado a ser muy alta con relación a otros países latinoamericanos, la incidencia de la extraedad escolar y la repitencia es por demás importante. Auguste, Echart y Franchetti (2008) aseguran que el país presenta serios problemas en el objetivo de la universalización de la educación secundaria, a pesar de la extensión de la obligatoriedad por ley en el 2006.

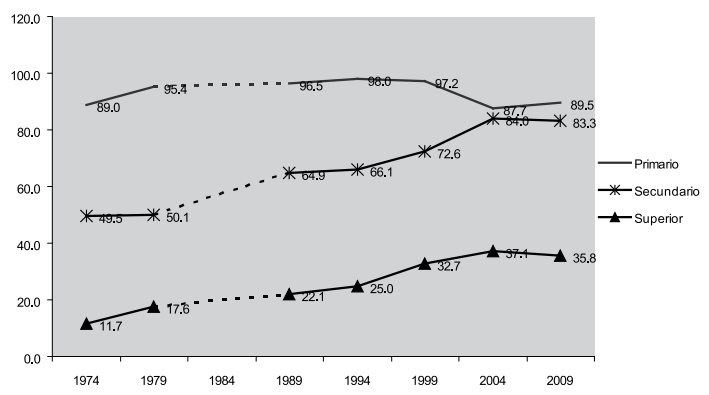

Figura $\mathrm{N}^{\circ} 2$ : Evolución de las tasas netas de escolarización para todos los niveles

Fuente: Elaboración propia con base en datos de la EPH (INDEC).

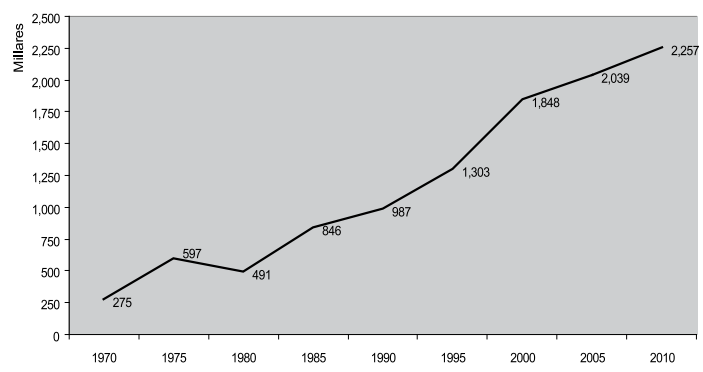

Figura $\mathrm{N}^{\circ}$ 3: Evolución de la matrícula total en el nivel superior

Fuente: Elaboración propia con base en Coraggio (2001) y Anuarios Estadísticos de la SPU y DINIECE (varios años).
La educación superior ha sido la de mayor crecimiento en el período analizado, multiplicándose la matrícula por diez a lo largo de 40 años (Figura 3) ${ }^{6}$. En los noventa, época de mayor repunte, el incremento se produjo principalmente por mejoras en las condiciones socioeconómicas a principios de la década; además de por la extensión de la educación media y las implicancias de un mayor desempleo y exigencias en el mercado laboral a partir de mediados de la década.

La inversión en educación posee mayores costos de oportunidad a nivel superior. Al mismo tiempo, se espera que sus rendimientos sean más elevados respecto de la educación media. Por ello, es de suponer que accederán aquellos individuos que puedan hacer frente a los costos presentes, a partir de la evaluación de los beneficios futuros. Las Tablas 3 a 8 muestran las tasas de escolarización para todos los niveles según grupo de deciles de ingreso. Por supuesto, son más elevadas para la educación terciaria y universitaria en el caso de los deciles superiores de ingreso, al mismo tiempo que se observa el aumento de las disparidades entre sectores bajos, medios $\mathrm{y}$ altos en términos de alcance de la educación según tasas brutas de matriculación. La brecha entre tasas netas y brutas es más amplia para los sectores de mayores ingresos, sugiriendo que la permanencia en el nivel de educación superior de los individuos más ricos es mayor que la de los individuos pertenecientes a los sectores sociales medios y aún mayor que la de los individuos más empobrecidos. $\mathrm{Al}$ mismo tiempo, factores como repitencia y sobreedad escolar en el nivel secundario parece afectar similarmente a todos los estratos sociales, con unos puntos más de incidencia en los sectores medios, en primer lugar, y bajos, en segundo término. 
Tabla 3

Tasas brutas de escolarización, total y según grupo de ingresos - Nivel primario ${ }^{(1)}$

\begin{tabular}{lcccccccc}
\hline & 1974 & $1979^{(2)}$ & 1984 & 1989 & 1994 & 1999 & 2004 & 2009 \\
\hline Total & 108.0 & 106.2 &. & 106.4 & 108.7 & 108.0 & 94.1 & 97.3 \\
Decil 1 a 4 & 106.1 & 110.3 &. & 107.2 & 108.7 & 109.6 & 95.8 & 99.9 \\
Decil 5 a 8 & 111.3 & 99.4 &. & 106.0 & 110.0 & 103.3 & 90.7 & 91.0 \\
Decil 9 y 10 & 121.7 & 98.9 &. & 100.7 & 103.0 & 99.0 & 87.1 & 92.4 \\
\hline
\end{tabular}

Fuente: Elaboración propia con base en datos de la EPH (INDEC)

(1) Corresponde a $1^{\circ}$ a $7^{\circ}$ grado de escuela primaria y $1^{\circ}$ a $7^{\circ}$ año modalidad EGB.

(2) Dato correspondiente a 1980.

Tabla 4

Tasas brutas de escolarización, total y según grupo de ingresos - Nivel secundario ${ }^{(1)}$

\begin{tabular}{lcccccccc}
\hline & 1974 & $1979^{(2)}$ & 1984 & 1989 & 1994 & 1999 & 2004 & 2009 \\
\hline Total & 62.7 & 64.7 &. & 78.8 & 81.9 & 95.7 & 120.4 & 116.6 \\
Decil 1 a 4 & 50.7 & 48.6 &. & 66.7 & 70.7 & 89.9 & 116.1 & 112.4 \\
Decil 5 a 8 & 74.6 & 76.0 &. & 93.4 & 91.6 & 112.8 & 129.7 & 125.8 \\
Decil 9 y 10 & 96.9 & 98.8 &. & 105.7 & 113.0 & 112.5 & 127.7 & 127.6 \\
\hline
\end{tabular}

Fuente: Elaboración propia con base en datos de la EPH (INDEC)

(1) Corresponde a $1^{\circ}$ a $5^{\circ}$ año de escuela secundaria, $8^{\circ}$ y $9^{\circ}$ año modalidad EGB y $1^{\circ}$ a $3^{\circ}$ año de polimodal.

(2) Dato correspondiente a 1980.

Tabla 5

Tasas brutas de escolarización, total y según grupo de ingresos - Nivel superior ${ }^{(1)}$

\begin{tabular}{lcccccccc}
\hline & 1974 & $1979^{(2)}$ & 1984 & 1989 & 1994 & 1999 & 2004 & 2009 \\
\hline Total & 22.0 & 31.2 &. & 40.1 & 43.3 & 58.5 & 75.9 & 74.5 \\
Decil 1 a 4 & 13.6 & 11.9 &. & 17.5 & 22.0 & 35.8 & 46.3 & 50.4 \\
Decil 5 a 8 & 21.6 & 25.6 &. & 38.9 & 45.3 & 95.3 & 104.2 & 101.2 \\
Decil 9 y 10 & 44.6 & 79.8 &. & 109.6 & 91.9 & 137.8 & 166.0 & 158.7 \\
\hline
\end{tabular}

Fuente: Elaboración propia con base en datos de la EPH (INDEC)

(1) Corresponde niveles universitario y terciario de grado.

(2) Dato correspondiente a 1980. 
Tabla 6

Tasas netas de escolarización, total y según grupo de ingresos - Nivel primario ${ }^{(1)}$

\begin{tabular}{lcccccccc}
\hline & 1974 & $1979^{(2)}$ & 1984 & 1989 & 1994 & 1999 & 2004 & 2009 \\
\hline Total & 89.0 & 95.4 &. & 96.5 & 98.0 & 97.2 & 87.7 & 89.5 \\
Decil 1 a 4 & 87.5 & 96.5 &. & 97.0 & 98.1 & 97.5 & 88.5 & 90.3 \\
Decil 5 a 8 & 92.3 & 93.8 &. & 97.0 & 98.2 & 96.1 & 86.0 & 87.2 \\
Decil 9 y 10 & 95.7 & 92.0 &. & 90.3 & 97.0 & 96.0 & 84.4 & 88.7 \\
\hline
\end{tabular}

Fuente: Elaboración propia con base en datos de la EPH (INDEC)

(1) Corresponde a $1^{\circ}$ a $7^{\circ}$ grado de escuela primaria y $1^{\circ}$ a $7^{\circ}$ año modalidad EGB.

(2) Dato correspondiente a 1980.

Tabla 7

Tasas netas de escolarización, total y según grupo de ingresos - Nivel secundario ${ }^{(1)}$

\begin{tabular}{lcccccccc}
\hline & 1974 & $1980^{(2)}$ & 1984 & 1989 & 1994 & 1999 & 2004 & 2009 \\
\hline Total & 49.5 & 50.1 & & 64.9 & 66.1 & 72.6 & 84.0 & 83.3 \\
Decil 1 a 4 & 41.1 & 37.9 & & 56.3 & 58.5 & 68.2 & 80.1 & 79.3 \\
Decil 5 a 8 & 57.7 & 58.3 & & 75.1 & 72.0 & 84.0 & 91.3 & 91.6 \\
Decil 9 y 10 & 73.8 & 77.4 & & 83.8 & 90.9 & 92.4 & 95.3 & 95.0 \\
\hline
\end{tabular}

Fuente: Elaboración propia con base en datos de la EPH (INDEC)

(1) Corresponde a $1^{\circ}$ a $5^{\circ}$ año de escuela secundaria, $8^{\circ}$ y $9^{\circ}$ año modalidad EGB y $1^{\circ}$ a $3^{\circ}$ año de polimodal.

(2) Dato correspondiente a 1980.

Tabla 8

Tasas netas de escolarización, total y según grupo de ingresos - Nivel superior ${ }^{(1)}$

\begin{tabular}{lcccccccc}
\hline & 1974 & $1979^{(2)}$ & 1984 & 1989 & 1994 & 1999 & 2004 & 2009 \\
\hline Total & 11.7 & 17.6 &. & 22.1 & 25.0 & 32.7 & 37.1 & 35.8 \\
Decil 1 a 4 & 8.7 & 7.1 &. & 12.5 & 14.1 & 21.4 & 24.9 & 26.8 \\
Decil 5 a 8 & 11.6 & 17.5 &. & 23.0 & 28.0 & 52.4 & 50.7 & 48.2 \\
Decil 9 y 10 & 19.2 & 36.1 &. & 47.8 & 44.4 & 62.2 & 65.7 & 55.8 \\
\hline
\end{tabular}

Fuente: Elaboración propia con base en datos de la EPH (INDEC)

(1) Corresponde niveles universitario y terciario de grado.

(2) Dato correspondiente a 1980. 


\section{Medidas de niveles educativos}

A partir de un proceso de recodificación de la información Recuperado de la EPH (años 2004 y 2009), se generaron variables para "cantidad de años de educación finalizados" y "nivel educativo". La primera de estas variables toma valores entre 0 y 19 , donde 0 corresponde a todos los individuos que no lograron finalizar al menos el primer año de la escuela primaria, 1 corresponde al primer grado de educación completo, 2 al segundo grado de la enseñanza primaria y así sucesivamente. El valor de 19 corresponde a "19 años de educación y más", lo cual incumbiría a niveles educativos de posgrado. La Tabla 9 muestra un resumen de los resultados para los períodos en que fue posible la estimación.

Tabla 9

Porcentaje de población según cantidad de años finalizados

\begin{tabular}{|c|c|c|c|c|c|}
\hline \multicolumn{3}{|c|}{ Población de 15 a 64 años } & \multicolumn{3}{|c|}{ Población de 25 a 64 años } \\
\hline $\begin{array}{l}\text { Años de } \\
\text { educación }\end{array}$ & 2004 & 2009 & $\begin{array}{l}\text { Años de } \\
\text { educación }\end{array}$ & 2004 & 2009 \\
\hline 0 & 0.91 & 0.71 & 0 & 1.13 & 0.87 \\
\hline 1 & 0.44 & 0.47 & 1 & 0.35 & 0.33 \\
\hline 2 & 0.21 & 0.13 & 2 & 0.21 & 0.14 \\
\hline 3 & 0.46 & 0.44 & 3 & 0.56 & 0.55 \\
\hline 4 & 1.22 & 0.89 & 4 & 1.57 & 1.07 \\
\hline 5 & 1.68 & 1.27 & 5 & 2.07 & 1.59 \\
\hline 6 & 1.26 & 1.18 & 6 & 1.53 & 1.38 \\
\hline 7 & 23.68 & 20.18 & 7 & 28.23 & 24.49 \\
\hline 8 & 0.29 & 0.34 & 8 & 0.02 & 0.03 \\
\hline 9 & 2.07 & 2.63 & 9 & 0.84 & 0.81 \\
\hline 10 & 2.76 & 2.82 & 10 & 2.45 & 2.23 \\
\hline 11 & 7.35 & 6.89 & 11 & 5.36 & 5.02 \\
\hline 12 & 28.90 & 30.48 & 12 & 28.59 & 31.08 \\
\hline 13 & 0.00 & 3.29 & 13 & 0.00 & 2.64 \\
\hline 14 & 4.29 & 3.49 & 14 & 1.50 & 1.26 \\
\hline 15 & 3.67 & 3.46 & 15 & 2.17 & 1.97 \\
\hline 16 & 10.42 & 10.95 & 16 & 11.00 & 11.93 \\
\hline 17 & 2.83 & 2.67 & 17 & 2.42 & 2.47 \\
\hline 18 & 7.33 & 7.52 & 18 & 9.73 & 9.84 \\
\hline 19 y más & 0.23 & 0.21 & 19 y más & 0.30 & 0.26 \\
\hline
\end{tabular}

Fuente: Elaboración propia con base en datos de la EPH, años 2004 y 2009 (INEDC) 
La población se presenta de forma heterogénea, con dos puntos de fuerte concentración en la distribución de la variable "años de educación": los 7 y los 12 años de escolarización finalizados. El primer número correspondería a quienes concluyeron el nivel primario, con alrededor de un $24 \%$ a $20 \%$ (según se trate del año 2004 o 2009) de la población. El segundo valor corresponde a una amplia proporción de población en edad de trabajar con nivel secundario finalizado, con entre un 29 y un $30.5 \%$ (2004 y 2009, respectivamente). Otro punto de relevancia corresponde a niveles superiores de educación, con entre un $10.4 \%$ y un $11 \%$ de la población que posee 16 años de educación completos. El mayor porcentaje en el grupo de 25 a 64 años de edad respecto del grupo de 15 a 64 años puede explicarse a partir de la inclusión en este segundo conjunto poblacional de individuos que se encuentran aún transitando el sistema educativo. Así, los años intermedios entre los 12 y los 16 años de educación muestran mayores porcentajes para el grupo de 15 a 64 años. Aunque no hay una variación significativa entre grupos etarios para el caso de los 12 años de educación, el grupo que incluye la fuerza laboral más joven muestra porcentajes sensiblemente mayores para los 11 años de educación.

Entre 2004 al 2009 se observan leves caídas en los porcentajes de los primeros grados educativos a favor de los años de educación a partir del octavo ciclo, suponiendo el incremento de la población con al menos el nivel secundario incompleto. Estas dos observaciones no pretenden indicar una tendencia, sino simplemente dar una idea de las situaciones observadas en dos momentos de tiempo. Por otra parte, cabe recordar la caída en la matriculación en el nivel primario analizada en el apartado anterior.

A partir de los años de educación efectivamente finalizados se estimaron los años promedio de educación del conjunto de la población. Este es el dato habitualmente reportado por las fuentes tales como OECD
(1998), Cohen y Soto (2007) o Barro y Lee (2010), mencionados anteriormente. En la Tabla 10 se exponen, de forma comparativa, los datos reportados por aquellos autores y los encontrados a partir de las estimaciones propias.

Son notorias las diferencias en los reportes. Los años promedio de escolarización establecidos por Barro y Lee (2010) y Cohen y Soto (2007) fueron inferiores a los obtenidos mediante la utilización de la $\mathrm{EPH}$. Recordando que los primeros autores obtenían el dato correspondiente a niveles educativos con base en la utilización de censos y estimando hacia atrás y hacia delante tales niveles ante la ausencia de observaciones y de acuerdo a las tasas de matriculación, a veces corrigiendo por repitencia o abandono escolar y multiplicando los niveles por la cantidad de años promedio que dicho nivel presupone, esta metodología podría volverse bastante inexacta, máxime cuando los niveles de no terminalidad son elevados. El método utilizado en este estudio se basa en la cantidad de años cumplidos exactamente y declarados por los individuos (independientemente de que hayan repetido algún año escolar). Por lo tanto, podría asegurarse una mayor precisión. Si esto fuera así, la literatura tradicional ha subestimado la medición del acervo educacional en el caso de Argentina.

Se evidencia un leve incremento del valor de dicha variable en el quinquenio señalado. Este indicador es mayor para el caso del grupo de 25 a 64 años, mostrando que la heterogeneidad en los niveles educativos entre individuos tiende a reducirse a partir de la consideración de los grupos de edad más joven, en quienes se evidencia el incremento reciente en niveles educativos.

La variable "nivel educativo" se construyó de acuerdo con los mayores niveles alcanzados y declarados por las personas como finalizados o no finalizados, y toma valores de 0 a 8 , según corresponda a "nunca asistió a ningún establecimiento educativo o asistió sólo a preescolar", "primario incompleto", "primario completo", 
"secundario incompleto", "secundario completo", "terciario/universitario incompleto", "terciario/universitario completo", "educación de postgrado incompleta" y "educación de postgrado completa", respectivamente.

Es interesante notar la evolución de la conformación de la fuerza laboral a lo largo del período 1974-2009 según los niveles educativos obtenidos. La Figura 4 evidencia un desplazamiento de la distribución de la población en edad laboral desde menores a mayores niveles educativos. Durante la década de 1970, se observa una sustancial disminución de la población con primaria incompleta y un acrecentamiento a partir del nivel secundario incompleto.

Tabla 10

Niveles educativos y años promedios de escolarización para Argentina, según fuente y año de relevamiento

\begin{tabular}{|c|c|c|c|c|}
\hline \multicolumn{5}{|c|}{ Porcentaje de población, según fuente y año relevado } \\
\hline & \multicolumn{4}{|c|}{15 a 64 años de edad } \\
\hline & \multicolumn{2}{|c|}{ Barro y Lee } & \multicolumn{2}{|l|}{ Rojas } \\
\hline & 2005 & 2010 & 2004 & 2009 \\
\hline \multicolumn{5}{|l|}{ Nivel educativo } \\
\hline No escolarizados & 2.9 & 2.4 & 0.9 & 0.7 \\
\hline Primario & 42.4 & 40.4 & 26.5 & 23.1 \\
\hline Secundario incompleto & 16.3 & 16.8 & 21.1 & 21.9 \\
\hline Secundario completo & 28 & 28.7 & 38.5 & 41.1 \\
\hline \multirow[t]{4}{*}{ Superior } & 9.9 & 12.2 & 14 & 14.2 \\
\hline & \multicolumn{4}{|c|}{25 a 64 años de edad } \\
\hline & \multicolumn{2}{|c|}{ Barro y Lee } & \multicolumn{2}{|l|}{ Rojas } \\
\hline & 2005 & 2010 & 2004 & 2009 \\
\hline \multicolumn{5}{|l|}{ Nivel educativo } \\
\hline No escolarizados & 3.6 & 2.9 & 1.1 & 0.8 \\
\hline Primario & 46.2 & 41.1 & 31.2 & 27.6 \\
\hline Secundario incompleto & 13.1 & 14.3 & 17.4 & 16.4 \\
\hline Secundario completo & 26.9 & 30.8 & 33.6 & 37.2 \\
\hline \multirow[t]{4}{*}{ Superior } & 10.2 & 10.9 & 16.7 & 18.0 \\
\hline & \multicolumn{4}{|c|}{15 a 64 años de edad } \\
\hline & \multicolumn{2}{|c|}{ Cohen y Soto } & \multicolumn{2}{|l|}{ Rojas } \\
\hline & 2000 & $2010(1)$ & 2004 & 2009 \\
\hline \multicolumn{5}{|l|}{ Nivel educativo } \\
\hline No escolarizados & 3.2 & 2 & 0.9 & 0.7 \\
\hline Primario & 56.2 & 53.3 & 26.5 & 23.1 \\
\hline Secundario incompleto & 15.7 & 16.7 & 21.1 & 21.9 \\
\hline Secundario completo & 18.4 & 20.6 & 38.5 & 41.1 \\
\hline Superior & 9.9 & 12.2 & 14 & 14.2 \\
\hline
\end{tabular}




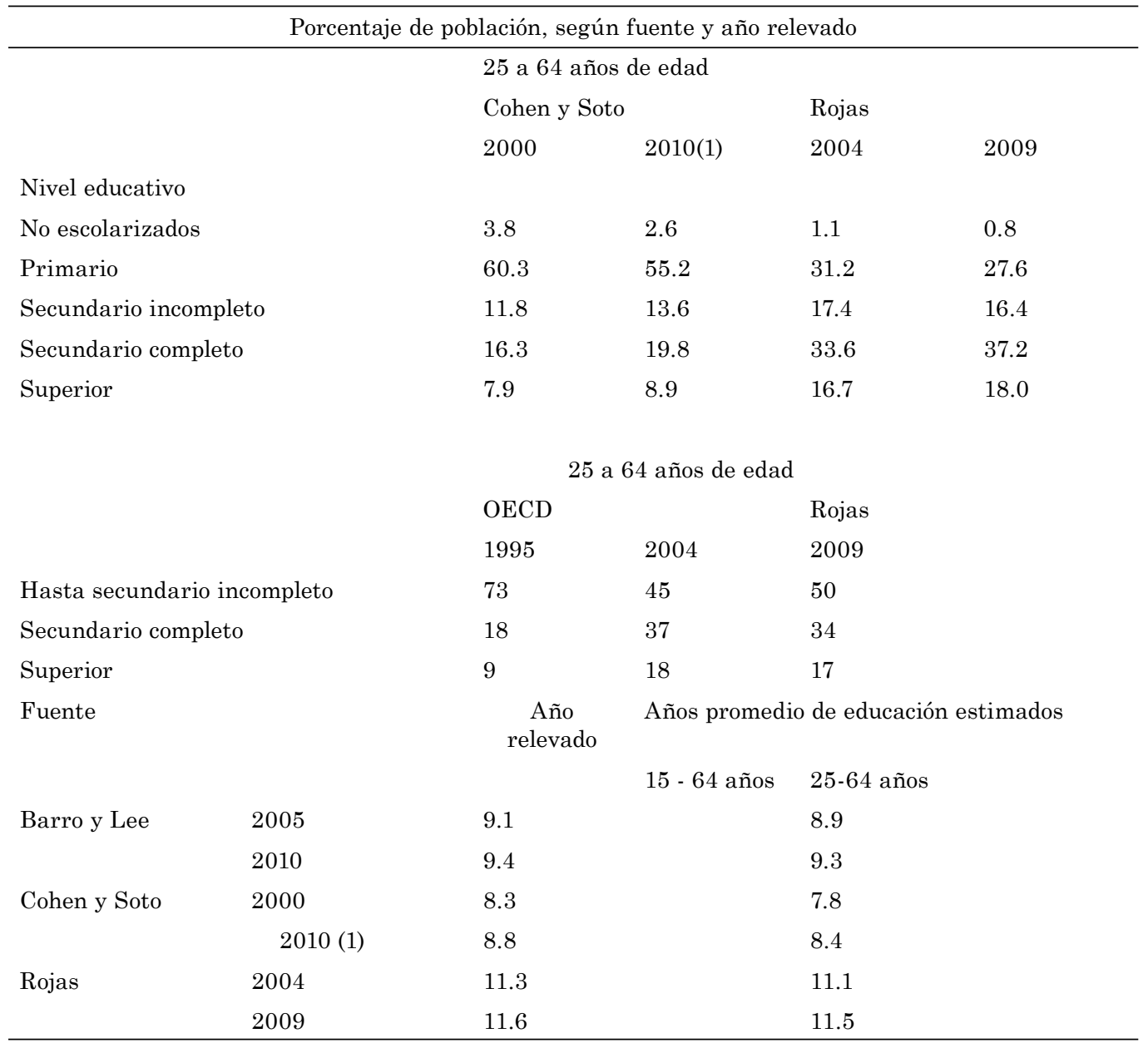

Fuente: Elaboración propia con base en Barro y Lee (2010), Cohen y Soto (2007), OECD (1998) y estimaciones propias (EPH - INDEC)

(1) Datos proyectados.

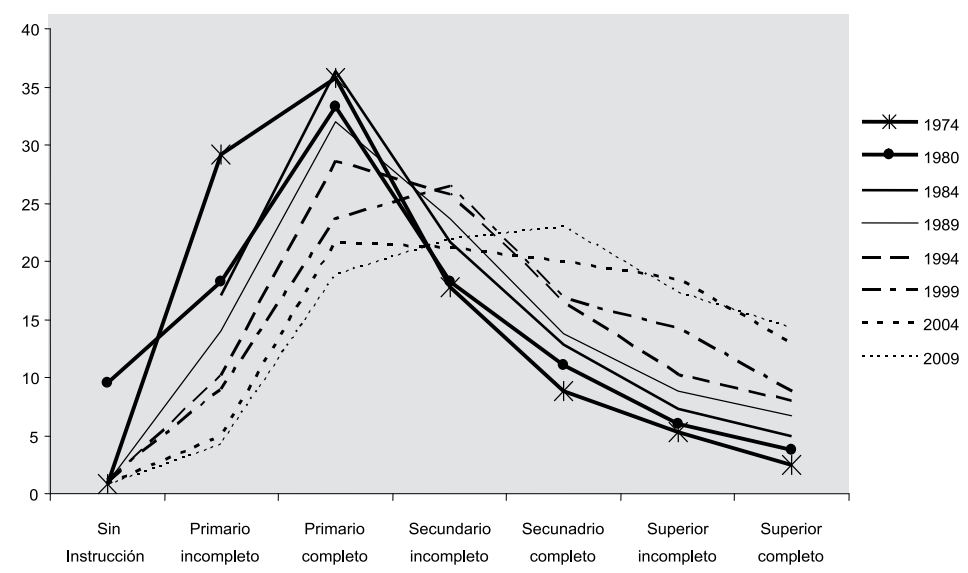

Figura $\mathrm{N}^{\circ}$ 4: Porcentaje de población de 15 a 64 años de edad según máximo nivel educativo alcanzado Fuente: Elaboración propia con base en datos de la EPH (varios años) (INDEC) 
Este proceso continuó durante la década de 1980, como se observa a partir del corrimiento sucesivo de las curvas graficadas para los años 1984 y 1989, aunque con menores reducciones en la injerencia de niveles primarios e incrementos proporcionales en los demás niveles. El pico superior de la distribución continuaba encontrándose en el nivel "primaria completa". A partir de entonces, es llamativo el cambio en las líneas graficadas. Las figuras para los años 1994 y 1999 exhiben dos fuertes concentraciones de la población en torno a los niveles primario completo y secundario incompleto.

Para los años 2004 y 2009 el aplanamiento de las curvas muestra una distribución más proporcional de la población en torno a los diferentes estamentos educativos, con amplios incrementos en los niveles secundario completo y superiores. En el caso de estas dos últimas observaciones, las cuales brindarían una representación aproximada del perfil poblacional reciente, los niveles "primario completo" y "secundario completo" concentran alrededor de un $40 \%$ de la población. En términos de esta variable, se evidencia cierto avance desde el 2004 y hasta el 2009 a partir de discretos incrementos en los niveles "secundario completo" y "superior completo", sobre todo al analizar el grupo de 25 años y más (Tabla 11).

Tabla 11

Porcentajes de población argentina según máximo nivel educativo alcanzado

\begin{tabular}{|c|c|c|c|c|c|c|c|c|}
\hline Nivel Educativo & 1974 & $1979^{(2)}$ & $1984^{(3)}$ & 1989 & 1994 & 1999 & 2004 & 2009 \\
\hline \multicolumn{9}{|c|}{ Población 15 a 64 años } \\
\hline Sin instrucción & 0.85 & 9.55 & & 0.95 & 0.92 & 1.13 & 0.88 & 0.70 \\
\hline Primario incompleto & 29.16 & 18.21 & 17.02 & 14.06 & 10.09 & 8.95 & 4.91 & 4.18 \\
\hline Primario completo & 35.72 & 33.25 & 36.37 & 32.04 & 28.53 & 23.65 & 21.60 & 18.88 \\
\hline Secundario incompleto & 17.71 & 18.24 & 21.60 & 23.59 & 25.78 & 26.45 & 21.12 & 21.87 \\
\hline Secundario completo & 8.83 & 11.06 & 12.82 & 13.80 & 16.44 & 16.79 & 19.99 & 22.90 \\
\hline Superior incompleto & 5.25 & 5.98 & 7.24 & 8.82 & 10.24 & 14.21 & 18.49 & 17.25 \\
\hline Superior completo & 2.49 & 3.71 & 4.95 & 6.74 & 8.00 & 8.81 & 13.00 & 14.20 \\
\hline Postgrado incompleto ${ }^{(1)}$ & & & & & & & 0.50 & 0.50 \\
\hline Postgrado completo ${ }^{(1)}$ & & & & & & & 0.26 & 0.33 \\
\hline \multicolumn{9}{|c|}{ Población 25 a 64 años } \\
\hline Sin instrucción & 0.96 & 9.38 & & 1.15 & 1.13 & 1.43 & 1.07 & 0.84 \\
\hline Primario incompleto & 33.23 & 21.67 & 20.62 & 16.92 & 12.11 & 11.02 & 5.90 & 4.89 \\
\hline Primario completo & 38.32 & 36.74 & 40.12 & 35.40 & 32.44 & 28.23 & 25.30 & 22.69 \\
\hline Secundario incompleto & 11.90 & 12.27 & 14.51 & 16.73 & 18.15 & 17.64 & 17.44 & 16.38 \\
\hline Secundario completo & 9.10 & 11.18 & 13.60 & 15.08 & 18.83 & 19.94 & 20.92 & 24.67 \\
\hline Superior incompleto & 3.39 & 4.26 & 5.08 & 6.36 & 7.00 & 9.45 & 12.71 & 12.49 \\
\hline Superior completo & 3.11 & 4.50 & 6.08 & 8.35 & 10.34 & 12.28 & 16.04 & 17.35 \\
\hline Postgrado incompleto ${ }^{(1)}$ & & & & & & & 0.28 & 0.25 \\
\hline Postgrado completo ${ }^{(1)}$ & & & & & & & 0.35 & 0.43 \\
\hline
\end{tabular}

Fuente: Elaboración propia con base en datos de la EPH (INDEC).

(1) Sólo a partir del año 2003, la encuesta permite la diferenciación de niveles de postgrado. Para los períodos anteriores, se incluye en nivel superior completo.

(2) Corresponde a datos de 1980.

(3) El nivel "sin instrucción" se reportó dentro del nivel "primario incompleto". 
Esto estaría en relación con la finalización durante estos años de la escolarización secundaria de muchos individuos afectados por las reformas educativas. También se observa en el quinquenio 2004-2009 una leve disminución del nivel de educación "superior incompleto" a favor del nivel "superior completo", condiciéndose con el máximo local establecido para los 16 años de educación reportados por la variable anteriormente analizada, lo que haría suponer que existe una mayor proporción de población con niveles superiores de educación en carreras de no más de 4 años de duración.

Mucha de la bibliografía citada agrupa los niveles inferiores al nivel secundario en una única categoría. Por ello, en la Tabla 10 anteriormente señalada se presenta un resumen de los resultados hallados, mediante la agrupación de niveles educativos según las categorías formuladas por OECD (1998), Cohen y Soto (2007) y Barro y Lee (2010) [7].

Dado que se trata de diferentes períodos muestrales y de diferentes metodologías, los datos no son estrictamente comparables. Sin embargo, las diferencias por niveles educativos son llamativas, al igual que en el caso de los años promedio de educación formal. Para el caso de los datos reportados por Barro y Lee (2010), y Cohen y Soto (2007), el nivel primario parece ser el punto de mayor concentración poblacional. Pero, al utilizar los datos directamente extraídos de la EPH, el nivel secundario se vuelve más relevante a la hora de explicar la conformación poblacional, según logros educativos. Asimismo, los niveles superior $\mathrm{y}$ secundario incompleto muestran algunos puntos porcentuales más de participación que en las estimaciones de los trabajos citados. Más allá de estas observaciones, la tendencia de incrementos a partir del nivel secundario en desmedro del nivel primario es claramente observada en todos los trabajos, mostrando sensibles mejoras en la evaluación en términos de niveles y años de educación.

\section{Consideraciones finales}

En este trabajo se intentó generar diferentes medidas del acervo educativo para Argentina con el objetivo de analizar la evolución reciente y los patrones actuales a lo largo del espacio poblacional. Al analizar los años o niveles de escolarización concluidos se retomaron las metodologías habituales como, por ejemplo, las desarrolladas por Barro y Lee (2010). La utilización de la $\mathrm{EPH}$, a pesar de reducir las observaciones a un espacio muestral, permitió la elaboración temporal exacta de tales indicadores, mostrando diferencias relevantes con otros trabajos que utilizan metodologías de aproximación para el caso de Argentina.

Por su parte, las trayectorias de los últimos años respecto de la acumulación de conocimientos y habilidades otorgadas por la educación formal parecen haber sido favorables, aunque cabe remarcar ciertas cuestiones fundamentales.

En primer lugar, las variaciones de las tasas de matriculación se corresponden ampliamente con los ciclos económicos, dejando desprotegidos a los sectores de menores ingresos, quienes se ven obligados a abandonar la escolarización en etapas de crisis imposibilitados de afrontar los costos. Clara muestra de ello es la enorme expansión surgida en el seno del sistema público de enseñanza a partir de las reformas educativas, junto con las mayores caídas en las tasas de matriculación en el sistema de gestión estatal en comparación con el sistema de educación privado ante períodos de dificultades económicas. La mayor estabilidad del alumnado en el sistema de gestión privada demuestra la posibilidad de una continua formación académica por parte de quienes pueden afrontar los costos de una educación pagada.

En segundo lugar, si bien el sistema se ha extendido enormemente en el período analizado, las brechas entre tasas netas y brutas de matriculación se han ampliado 
de la misma forma, demostrando mayores pérdidas en términos de extraedad, repitencia y abandono escolar, particularmente en la educación secundaria y superior. Si bien no se ha realizado un análisis de calidad educativa (lo cual queda fuera de los propósitos de este trabajo de investigación), sería interesante analizar la evolución en estos términos dada la ampliación que sufrió el sistema a lo largo del período analizado, al tiempo que se incrementaron las diferencias entre tasas netas y brutas de matriculación. La masificación del sistema educativo podría haber deteriorado los procesos de enseñanza y dificultado el aprendizaje por parte del alumnado, incrementándose las tasas de repitencia, extraedad y retraso escolar y, por ello, la diferencia entre tasas brutas y netas.

A pesar de que Argentina lidera las estadísticas latinoamericanas en educación, aún existe una amplia cantidad de individuos con bajos niveles relativos de conocimientos enmarcados en un contexto de profundas dualidades socioeconómicas. El $20 \%$ de la población joven no accede a la escuela secundaria, logrando concluir el ciclo menos de la mitad de quienes acceden. En los niveles secundario y superior, las menores tasas netas se registran en los deciles más bajos de la distribución. De existir una relación positiva entre los niveles educacionales alcanzados y las posibilidades de obtención de empleo e ingresos a futuro [8], el menor alcance educativo de los adolescentes pertenecientes a los estratos más empobrecidos de la población reduciría las posibilidades de movilidad social y aseguraría la retroalimentación de las desigualdades iniciales de ingresos.

Rojas y Formichella (2011) señalan algún reconocimiento de la situación por parte de Estado Nacional a partir de las últimas reformas iniciadas en 2006, cuando "(l) a consecución de la equidad se vuelve central en el discurso de cada nueva norma, las políticas sociales se tornan en una mayor focalización, e incluso el gasto por alumno se profundiza" (p. 34).
Políticas como el Programa Nacional de Inclusión Educativa, el cual abarca diferentes subprogramas, tales como Volver a la Escuela (para niños de 6 a 14 años de edad que hubieran abandonado la educación primaria) y Todos a Estudiar (para niños de entre 11 y 18 años de edad que no hubieran finalizado la educación media) poseen claras especificaciones tanto pedagógicas (en el caso de Volver a la Escuela, que incluye objetivos educacionales y tutores que acompañan a los beneficiarios de las becas en el proceso de aprendizaje) como de redireccionamiento del gasto público.

El problema de inequidad, no obstante, es aún más amplio y complejo. La crisis de 2001 produjo la rotura del tejido social argentino. La desigualdad medida por el coeficiente de Gini trepó a 0.53 puntos, mientras que más del $50 \%$ de la población se hallaba bajo la línea de pobreza y alrededor de un $20 \%$ era indigente, luego de que el desempleo llegara a los veinte puntos porcentuales durante la década de 1990 (London y Rojas, 2010). La implementación de planes sociales tendientes a reducir los problemas de desempleo, pobreza y desigualdad también rozó la problemática educativa. Si bien en el caso de la educación primaria las mayores diferencias entre tasas netas y brutas se observan, en este trabajo, para los deciles 1 a 4, las más bajas tasas netas de escolarización corresponden a los deciles medios, los más perjudicados luego de la crisis mencionada. La explicación de la presencia de mayores tasas brutas de escolarización primaria en el grupo poblacional de menores ingresos es, precisamente, la existencia de políticas que poseen como objetivo indirecto la retención de los niños pertenecientes a estos estratos en la escuela, no ya sólo por fines educacionales sino también de contención y complementación de diferentes tipologías de planes sociales puestos en marcha desde el 2003 [9].

Si la fracción de población más empobrecida no logra acceder a los niveles medio y superior de educación para quebrar el 
problema intergeneracional de bajos niveles de ingresos, al tiempo que se produce un desmejoramiento del acceso a la educación primaria de los estratos sociales medios, la consecuencia será la persistencia o, incluso, incremento de las desigualdades. A fin de evitar el agravamiento de esta situación, debería comenzarse por una revisión y profundización en la focalización de las políticas ya existentes, siendo estas tomadas como parte de un plan integral de inclusión social. Mantener la regularidad en la escuela primaria de los niños a cargo (requisito para la obtención de planes sociales de ayuda asistencial o laboral para el grupo adulto de la población) implica una mínima asistencia a la institución no cumpliéndose, la mayoría de las veces, con los objetivos pedagógicos. En tal caso, los programas de focalización mencionados más arriba deberían cumplir más satisfactoriamente los objetivos de enseñanza, mientras que las metas educativas no deberían confundirse con los requisitos impuestos para el otorgamiento de otros tipos de planes sociales con el objetivo de no conducir a incentivos incorrectos a la hora de priorizar la educación de los integrantes más jóvenes de la sociedad.

\section{Notas}

1 La EPH tiene por objeto relevar características sociodemográficas y socioeconómicas de la población argentina. En este momento, la EPH cubre un espacio de 31 aglomerados urbanos (ciudades de más de 100 mil habitantes) y un área urbano-rural (INDEC, 2003). La encuesta se subdivide en dos partes: características de las personas, en donde se indaga respecto de los niveles y años de educación, situación laboral e ingresos personales, entre otras cosas; además, características de los hogares, en donde se pregunta acerca de las características habitacionales de la vivienda y la composición familiar. La EPH se realiza en el país desde 1974, con algunas interrupciones temporales, con frecuencia semestral y de manera puntual (ondas mayo y octubre) hasta el 2003, y de manera continua y frecuencia trimestral desde entonces.
2 La principal crítica que podría recibir el uso de estas encuestas es que el universo abarca únicamente zonas urbanas, quedando relegadas las zonas rurales del país. Sin embargo, es importante remarcar que para el caso de Argentina, la población urbana representa alrededor del $90 \%$ de la población total. En aquellos casos en donde la población rural es significativa, las encuestas suelen considerar zonas rurales. De aplicarse la metodología a otros países, debería tenerse en cuenta este fenómeno.

3 La reforma educativa acaecida en Argentina durante los años noventa estableció la heterogeneidad institucional en el sistema, coexistiendo en paralelo durante varios años el sistema tradicional de niveles primario y secundario con el sistema designado por niveles de Educación General Básica (EGB) y Polimodal. Para el año 2007, se retoma el sistema de educación dividido en ciclos primario y secundario. Por tal motivo y a fin de mantener la homogeneidad a lo largo del trabajo, al hablar del ciclo primario, se hace referencia a los primeros 7 años de educación básica (esto es, ciclo primario o EGB 1 y 2, según sea el sistema vigente), mientras que para el cálculo de tasas y niveles de escolarización secundaria se consideraron los 5 o 6 años restantes de educación básica (EGB 3 y Polimodal o escuela secundaria, en donde las modalidades técnicas suelen poseer 6 años de enseñanza). El sistema de información del Ministerio de Educación argentino es reformado a partir de 1996, no encontrándose datos publicados con anterioridad a esa fecha.

5 Según Auguste, Echart y Franchetti (2008), la meta de universalización de la educación primaria ya había sido cubierta por Argentina a inicios de los setenta, siendo el primer país de la región en obtener dicho logro.

Respecto de la educación superior, la última mitad del siglo XX fue testigo de la extensión de los sistemas de educación superior en todos los países de Latinoamérica. Para dar una idea de la masificación ocurrida en los sistemas de educación superior universitario y no universitario en Argentina, la cantidad de alumnos insertos en ambos ámbitos se incrementó de 275.000 en 1970 a 1,51 millones en el año 2000 (Coraggio, 2001).

7 Los trabajos en esta materia suelen basarse para establecer los niveles de educación en la Clasificación Internacional Normalizada de la Educación (CINE), establecida por la UNESCO en 1975. La misma fue formulada como "un instrumento idóneo para el acopio, compilación y presentación de estadísticas de educación en los distintos países y también en 
un plano internacional" (UNESCO, 2006, p. iii). Barro y Lee (2010) y Cohen y Soto (2007) toman las seis categorías: "ninguna educación formal", "primario incompleto", "primario completo", "primer ciclo del secundario", "segundo ciclo del secundario" y "terciario". Finalmente, para la presentación de los datos agregan las dos categorías para educación primaria y secundaria, diferenciando esta última por nivel completo e incompleto. Según la CINE, el primer ciclo de la educación secundaria se corresponde con el segundo ciclo de la educación básica (esto es, EGB 3 para el caso de Argentina). Por lo tanto, al incluir la población con $8^{\circ}$ y $9^{\circ}$ año en el nivel de educación secundaria, se lleva a cabo un agrupamiento igual al realizado por Barro y Lee (2010) y Cohen y Soto (2007).

Un buen análisis de los retornos sobre la inversión en educación se detalla en Carlson (2002) y Rojas (2011). En estos trabajos se concluye que los diferenciales de rendimientos sobre la inversión en educación en términos salariales se acentúan a partir de la obtención de escolarización secundaria completa y, más aún, del nivel de educación superior.

Entre otros, pueden mencionarse los casos del Plan Jefes y Jefas de Hogar, implementado en el 2003, y el Plan de Asignación Universal por Hijo, iniciado en el 2009. En el primer caso, se trata de un programa de empleo estatal que requiere la regularidad de los hijos menores de 18 años en el sistema educativo para ser otorgado. En el segundo caso, se trata de un subsidio de seguridad social cuyos beneficiarios son las personas no inscriptas en el sistema formal de empleo y que posean a cargo hijos menores de edad. También requiere de la asistencia de los menores a la escuela como requisito de otorgamiento.

\section{Referencias bibliográficas}

Auguste, S.; Echart, M y Franchetti, F. (2008). The Quality of Education in Argentina. Washington D.C.: Inter-American Development Bank. Recuperado de http://www.iadb.org/res/laresnetwork/ files/pr294finaldraft.pdf

Barro, R. y Lee, J.W. (1993). "International comparisons of educational attainment". Journal of Monetary Economics, 32, pp. 363-94. doi:10.1016/0304-3932(93)90023-9
Barro, R. y Lee, J.W. (1996). "International measures of schooling years and schooling quality". American Economic Review Papers and Proceedings, 86(2), pp. 218-23. Recuperado de http://www.jstor.org/ stable/10.2307/2118126

Barro, R. y Lee, J.W. (2000). "International data on educational attainment. Updates and implications”. NBER Working Paper Series, 7911, Cambridge, M.A.: National Bureau of Economic Research. Recuperado de http://www.nber.org/papers/w7911

Barro, R. y Lee, J.W. (2010). "A new data set of educational attainment in the world”. NBER Working Paper Series, 15902, Cambridge, M.A.: National Bureau of Economic Research. Recuperado de http://www.nber.org/ papers/w15902

Brunner, J.J. (2005). Tendencias recientes de la Educación Superior a nivel internacional: marco para la discusión sobre procesos de aseguramiento de la calidad. Documento de trabajo. Viña del Mar: Universidad Adolfo Ibáñez.

Brunner, J.J. y Bricall, J.M. (2000). Universidad siglo XXI. Europa $y$ América Latina, regulación y financiamiento. Documentos Columbus sobre Gestión Universitaria. París: Programa Columbus. Recuperado de http://www.columbus-web.org/index. php?option=com_content\&task=view \&id=100\&Itemid $=182$

Carlson, B. (2002). "Educación y mercado de trabajo en América Latina". Revista de la CEPAL, 77, pp. 123-141.

Cohen, D. y Soto, M. (2007). "Growth and human capital: Good data, good results". Journal of Economic Growth, 12(1), pp. 51-76. doi: 10.1007/ s10887-007-9011-5

Coraggio, J.L. (2001). Construir Universidad en la Adversidad, Desafios de la Educación Superior en América Latina. II Reunión 
de Ministros de Educación de las Américas en el Ámbito del CIDI (Consejo Interamericano para el Desarrollo Integral). Punta del Este: Unidad de Desarrollo Social y Educación de la OEA.

De la Fuente, A. y Doménech, R. (2006). "Human capital in growth regressions: How much difference does data quality make?" Journal of the European Economic Association, 4(1), pp.1-36. doi: 10.1162/jeea.2006.4.1.1

DINIECE, (varios años). Anuario Estadistico, Buenos Aires: Ministerio de Educación. Recuperado de http://diniece. me.gov.ar/index.php?option $=\mathrm{com}_{-}$ content\&task=category\&sectionid= $2 \&$ id=8\&Itemid=19

Formichella, M.M. y Rojas, M. (2007). "El proceso de descentralización de la educación en Argentina. Un caso: la Provincia de Buenos Aires", en Perspectivas de la educación en América Latina (pp. 167-188). Quito: Ministerio de Cultura de Ecuador y FLACSO Ecuador Eds.

INDEC, (2003). La Encuesta Permanente de Hogares de Argentina. Buenos Aires: INDEC. Recuperado de http://www. indec.gov.ar/nuevaweb/cuadros/4/ Metodologia_EPHContinua.pdf

INDEC, (2010). Glosario. Buenos Aires: INDEC. Recuperado de http://www. indec.gov.ar/glosario/glosario_glosario.asp

INDEC, (varios años). Bases Usuarias de la Encuesta Permanente de Hogares. Buenos Aires: INDEC. Recuperadas en http://www.indec.gov.ar/

Kyriacou, G. (1991). Level and growth effects of human capital, a cross-country study of the convergence hypothesis. Economic Research Report, C.V. Starr Center for Applied Economics, New York, N.Y.: New York University.

London, S. y Rojas, M. (2010). "El fenómeno de la desigualdad en Argentina", en Accinelli E. y Salas, O. Editores,
Crecimiento y Distribución del Ingreso en América Latina (pp: 109131). México D.F.: Astra Ediciones.

Nehru, V., Swanson, E. y Dubey, A. (1995). "A new database on human capital stocks in developing and industrial countries: Sources, methodology and results". Journal of Development Economics, 46(2), pp. 379-401.

OECD (1998). Education At A Glance: OECD Indicators. París: Centre for Educational Research and Innovation.

Psacharopoulos, G. y Arriagada, A.M. (1986). "The educational composition of the labor force: An international comparison". Intemational Labor Review, 125(5), pp.561-574.

Psacharopoulos, G. y Arriagada, A.M. (1992). "The educational composition of the labor force: An intemational update". ER_EE Background Paper Series, PHREE/92/49. Education and Employment Division, Population and Human Resources Department, Washington D.C.: The World Bank.

Rojas, M. (2011). Planificación educativa para el desarrollo: influencia de variables económicas y sociales sobre la formación de capital humano. (Tesis inédita de doctorado). Universidad Nacional del Sur, Bahía Blanca, Argentina.

Rojas, M. y Formichella, M.M. (2011). Cambios en el Sistema Educativo Argentino. El proceso de descentralización y la posterior reforma. Barcelona: Editorial Académica Española (Mimeo)

Secretaría de Políticas Universitarias (varios años). Anuario Estadístico, Buenos Aires: SPU. Recuperado de http://www.me.gov.ar/spu/ Publicaciones/publicaciones.html

UNESCO (2006). Clasificación internacional normalizada de la educación, Montreal: UIS - UNESCO. Recuperado de http://unesdoc.unesco. org/images/0014/001470/147002s.pdf 
